\title{
Lesions of Acetylcholine Neurons in Refractory Epilepsy
}

\author{
Masaharu Hayashi, ${ }^{1}$ Keisuke Nakajima, ${ }^{1}$ Rie Miyata, ${ }^{1}$ Naoyuki Tanuma, ${ }^{1}$ and Tohru Kodama ${ }^{2}$ \\ ${ }^{1}$ Department of Brain Development and Neural Regeneration, Tokyo Metropolitan Institute of Medical Science, Tokyo 156-8506, Japan \\ ${ }^{2}$ Department of Psychophysiology, Tokyo Metropolitan Institute of Medical Science, Tokyo 156-8506, Japan
}

Correspondence should be addressed to Masaharu Hayashi, hayashi-ms@igakuken.or.jp

Received 13 March 2012; Accepted 8 May 2012

Academic Editor: F. G. Wouterlood

Copyright (C) 2012 Masaharu Hayashi et al. This is an open access article distributed under the Creative Commons Attribution License, which permits unrestricted use, distribution, and reproduction in any medium, provided the original work is properly cited.

\begin{abstract}
We have examined brainstem lesions in patients with refractory epilepsy disorders, including West syndrome (WS), LennoxGastaut syndrome (LGS), and dentatorubral-pallidoluysian atrophy (DRPLA). Acetylcholinergic neurons (AchNs) in the pedunculopontine tegmental nucleus $(\mathrm{PPN})$ are involved in mental development, and disruption of neuronal nicotinic acetylcholine receptors can lead to epilepsy. In order to investigate the involvement of lesions of AchNs in refractory epilepsy, we performed immunohistochemical analyses of AchNs in the PPN in autopsy cases who had a past history of WS and/or LGS and in DRPLA cases who showed progressive myoclonic epilepsy. In addition, we performed a preliminary quantification of the levels of acetylcholine, neuropeptides, and monoamine metabolites in the cerebrospinal fluid (CSF) of patients with WS and benign convulsions associated with mild gastroenteritis $(\mathrm{CwG})$. In the PPN analysis, the total number of neurons and the number of AchNs were reduced in WS/LGS and WS cases, while DRPLA cases showed a decrease in the number and percentage of AchNs. In the CSF analysis, WS patients demonstrated a reduction in the levels of inhibitory neuropeptides, while CwG patients showed increased levels of acetylcholine and decreased levels of serotonin metabolites. These data suggest the possible involvement of lesions of AchNs in WS and DRPLA.
\end{abstract}

\section{Introduction}

West syndrome (WS), which consists of tonic spasms, psychomotor developmental delay, and characteristic electroencephalography changes involving hypsarrhythmia, can have various causes, including congenital brain anomalies and sequelae after perinatal hypoxic and ischemic encephalopathy (HIE) [1]. Lennox-Gastaut syndrome (LGS) usually develops subsequently in patients with symptomatic WS. Although many anticonvulsants have been developed and treatments with adrenocorticotropins and ketogenic diets have been elaborated, epileptic seizures tend to be resistant to anticonvulsant therapies, and the mental prognosis remains poor in most patients with WS and/or LGS [2]. Progressive myoclonus epilepsy (PME) is another entity of refractory epilepsy, and the etiologies of PME include various disorders, such as neuronal ceroid-lipofuscinosis and UnverrichtLundborg disease [3]. Dentatorubral-pallidoluysian atrophy (DRPLA), which is an autosomal dominant neurodegenerative disorder, is one of the representative diseases that cause PME in Japan [4]. DRPLA is classified into 3 major types: (1) juvenile DRPLA with onset before the age of 20 and PME, (2) early adult type with onset between 20 and 40 years of age, ataxia, choreoathetosis, dementia, and PME, and (3) late adult type with a diagnosis made after 40 years of age often developing ataxia and dementia but not PME [5]. PME is refractory to anticonvulsant therapies in patients with DRPLA, and almost all patients have poor seizure and mental prognoses [4]. In order to clarify the epileptogenesis and contribute to the development of new treatments of refractory epilepsy, we have examined lesions of monoaminergic neurons in the brainstem of autopsy cases of WS/LGS and DRPLA [6, 7].

Acetylcholinergic neurons (AchNs) in the nucleus basalis of Meynert (NbM) and the pedunculopontine tegmental nucleus (PPN) are involved in mental development and learning abilities [8]. Lesions of the AchN system have been observed in developmental brain disorders, such as Down syndrome and Rett syndrome [9]. The PPN, which is in the lower midbrain, contains AchNs, catecholaminergic neurons 
(CANs), GABAergic interneurons (GABAis), and nonacetylcholinergic and noncatecholaminergic neurons and has afferent and efferent connections to the cerebrum and spinal cord [10]. The cholinergic innervation from the PPN to the thalamus and pons is involved in the generation of muscle tone and rapid eye movement sleep, and the PPN is believed to be a part of the mesencephalic locomotor region. In controls, there is an age-dependent change in the percentages of AchNs and CANs in the PPN. The percentages of AchNs and CANs are $20 \%$ to $30 \%$ and $10 \%$ to $15 \%$ in the PPN in children and young adults, respectively, while the percentage of AchNs decreases to 5\% to $10 \%$ and that of CANs increases to $20 \%$ to $30 \%$ in middle-aged and elderly subjects [11]. We have performed an immunohistochemical analysis and clarified the selective lesioning of AchNs in the PPN and/or the NbM in patients with perinatal disorders [11], PraderWilli syndrome [12], and xeroderma pigmentosum [13], which is caused by inherited disturbances in nucleotide excision repair. However, neuronal nicotinic acetylcholinergic receptors (nAChRs), which mediate cholinergic signaling, are involved in the pathogenesis of a number of neurological disorders [14]. The identification of mutations in the neuronal $\alpha 4 \beta 2 \mathrm{nAChRs}$ is well known to be a key for the understanding of the pathogenesis of autosomal dominant nocturnal frontal lobe epilepsy (ADNFLE) [15]. Nevertheless, lesions of AchNs have never been examined in either autopsy brains or the cerebrospinal fluid (CSF) of patients suffering from refractory epilepsy.

In order to investigate the involvement of the acetylcholinergic neuronal system in epileptogenesis, we performed an immunohistochemical analysis of AchNs, CANs, and GABAis in the PPN in autopsy cases with a history of WS and/or LGS and DRPLA. In addition, we preliminarily evaluated the CSF levels of acetylcholine, neuropeptides, and monoamine metabolites in patients with WS and benign convulsions associated with mild gastroenteritis (CwG) (disease controls).

\section{Materials and Methods}

2.1. Subjects in the Pathological Analysis. The clinical subjects included 6 cases with a history of both WS and LGS (WS/LGS cases), 6 cases with a history of only WS (WS cases), and 7 cases with clinically and genetically confirmed juvenile and early adult types of DRPLA showing PME in addition to 6 controls with no pathological changes in the central nervous system; subjects were aged from 4 months to 40 years (Table 1). Congenital brain malformation was the etiology in 4 of 6 WS/LGS cases and in 5 of 6 WS cases. The ethical committee of the Tokyo Metropolitan Institute of Medical Science approved this study, and the family of each subject provided informed consent for the postmortem analysis.

2.2. Immunohistochemistry. Brains were fixed in a buffered formalin solution. Each formalin-fixed brain was cut coronally and then embedded in paraffin. Six $\mu \mathrm{m}$ thick serial sections were cut from the lower midbrain, including the PPN. After microwave antigen retrieval, each section was
TABLE 1: Summary of the subjects in the pathological analysis.

\begin{tabular}{|c|c|}
\hline Age/sex & Cause of death \\
\hline \multicolumn{2}{|l|}{ Control } \\
\hline $4 \mathrm{~m} / \mathrm{male}$ & Peritonitis \\
\hline $1 \mathrm{y} / \mathrm{male}$ & Malignant lymphoma \\
\hline $6 \mathrm{y} / \mathrm{male}$ & Acute leukemia \\
\hline $22 \mathrm{y} / \mathrm{male}$ & DIC \\
\hline $29 \mathrm{y} / \mathrm{male}$ & Migraine \\
\hline \multirow[t]{2}{*}{$38 \mathrm{y} /$ female } & Heart failure \\
\hline & Etiology \\
\hline \multicolumn{2}{|c|}{ West syndrome/Lennox-Gastaut syndrome } \\
\hline $5 \mathrm{y} /$ female & Lissencephaly \\
\hline $8 \mathrm{y} / \mathrm{male}$ & Lissencephaly \\
\hline $10 \mathrm{y} /$ female & Lissencephaly \\
\hline $13 \mathrm{y} / \mathrm{male}$ & Perinatal HIE \\
\hline $18 \mathrm{y} / \mathrm{male}$ & Perinatal HIE \\
\hline $21 \mathrm{y} / \mathrm{male}$ & Lissencephaly \\
\hline \multicolumn{2}{|c|}{ West syndrome only } \\
\hline $5 \mathrm{y} /$ female & Microdysgenesis \\
\hline $13 \mathrm{y} /$ female & Microdysgenesis \\
\hline $16 \mathrm{y} / \mathrm{male}$ & Heterotopia \\
\hline $20 \mathrm{y} / \mathrm{male}$ & Perinatal HIE \\
\hline $24 \mathrm{y} / \mathrm{male}$ & Porencephaly \\
\hline \multirow[t]{2}{*}{$26 \mathrm{y} / \mathrm{Male}$} & Microdysgenesis \\
\hline & Disease type \\
\hline \multicolumn{2}{|c|}{ Dentatorubral-pallidoluysian atrophy } \\
\hline 16 y/female & Juvenile \\
\hline $17 \mathrm{y} / \mathrm{male}$ & Juvenile \\
\hline $21 \mathrm{y} / \mathrm{male}$ & Juvenile \\
\hline $24 \mathrm{y} / \mathrm{male}$ & Juvenile \\
\hline $27 \mathrm{y} / \mathrm{male}$ & Juvenile \\
\hline $39 \mathrm{y} /$ female & Early adult \\
\hline $40 \mathrm{y} / \mathrm{male}$ & Early adult \\
\hline
\end{tabular}

m: months; y: years; DIC: disseminated intravascular coagulation; HIE: hypoxic ischemic encephalopathy.

treated with mouse monoclonal antibodies to microtubuleassociated protein 2 (MAP2; 1 : 100, Upstate Cell Signaling Solutions, Billerica, MA, USA), acetylcholinesterase (AchE; Affinity Bioreagents, Inc, Golden, CO, USA), tyrosine hydroxylase (TH; Affinity Bioreagents, Inc), calbindin-D28K (CD; Novocastra Laboratories, Newcastle upon Tyne, UK), and glial fibrillary acidic protein (GFAP; Nichirei Corporation, Tokyo, Japan) at the following concentrations: $1: 1$ (GFAP), $1: 100$ (MAP2 and CD), 1:250 (AchE), and $1: 400(\mathrm{TH})$. Antibody binding was visualized using the avidin-biotin-immunoperoxidase complex method (Nichirei Corporation) according to the manufacturer's protocol. No staining was detected in the sections in the absence of antibody.

2.3. Quantitative Evaluation in the PPN and Data Analysis. The PPN was identified dorsolateral to the rostral superior cerebellar peduncle and the medial lemniscus in the lower 
TABLE 2: Summary of the subjects in the analysis of cerebrospinal fluid.

\begin{tabular}{lc}
\hline Age/sex & Etiology \\
\hline Control & \\
$1 \mathrm{~m} /$ female & Upper respiratory infection \\
$1 \mathrm{~m} /$ female & Upper respiratory infection \\
$2 \mathrm{~m} / \mathrm{male}$ & Viral gastroenteritis \\
$2 \mathrm{~m} / \mathrm{male}$ & Viral gastroenteritis \\
$6 \mathrm{~m} /$ female & Upper respiratory infection \\
Benign convulsion with mild gastroenteritis \\
$8 \mathrm{~m} /$ male & Viral gastroenteritis \\
$1 \mathrm{y} /$ female & Viral gastroenteritis \\
$2 \mathrm{y} / \mathrm{male}$ & Viral gastroenteritis \\
$2 \mathrm{y} / \mathrm{male}$ & Viral gastroenteritis \\
$2 \mathrm{y} / \mathrm{male}$ & Viral gastroenteritis \\
\hline & Disease type prognosis \\
\hline West syndrome & Cryptogenic \\
$5 \mathrm{~m} /$ female & Normal development \\
$6 \mathrm{~m} /$ female & Symptomatic \\
$8 \mathrm{~m} /$ female & Normal development \\
& Cryptogenic \\
\hline
\end{tabular}

m: months; y: years.

midbrain, as previously reported [11]. The PPN is composed of clusters of moderately large neurons (pars compacta) and the more widespread pars dissipata in the rostral and medial regions. In the pars compacta of the PPN, the number of neurons that were immunoreactive for MAP2, AchE, $\mathrm{TH}$, and $\mathrm{CD}$ was determined after the manual labeling of appropriate neurons with nucleoli in 2 serial sections, and the mean value was calculated. The percentages of neurons that were immunoreactive for AchE, TH, and CD relative to those that were immunoreactive for MAP2 were also calculated. All results are presented as the mean (SD). Kruskal-Wallis tests were used to analyze the differences in the data among controls and cases of WS/LGS and WS for a quantitative evaluation of immunoreactive cells. Comparisons of data between DRPLA cases and controls were analyzed by nonparametric Mann-Whitney $U$ tests.

2.4. Subjects in the Analysis of the CSF. Clinical subjects included 3 infants with clinically and electrophysiologically confirmed WS, 5 patients with CwG, and 5 normal controls with acute viral infections who lacked neurological abnormalities at the follow-up examination; subjects were aged from 1 month to 2 years (Table 2). CwG is a situationrelated seizure disorder that is characterized by an age at onset of 1 to 3 years, clustering seizures, normal interictal electroencephalography and neuroimaging, and favorable outcomes, as previously reported in Japan [16]. The CSF samples were obtained from 8 epileptic patients before the start of anticonvulsant therapy and from 5 normal controls at the time of examinations of fever. They were immediately frozen and stored in a $-80^{\circ} \mathrm{C}$ freezer until the measurement. The ethical committee of the Tokyo Metropolitan Institute of Medical Science approved this analysis, and the family of each subject provided informed consent for the utilization of CSF samples.

2.5. Quantitative Evaluations of Acetylcholine, Neuropeptides, and Monoamine Metabolites in the CSF and Data Analysis. Concentrations of acetylcholine, neuropeptides, including aspartate, glutamate, glycine, and GABA, and monoamine metabolites, such as 3-methoxy-4-hydroxyphenylethylglycol (MHPG), homovanillic acid (HVA), and 5-hydroxyindoleacetic acid (5-HIAA), in the CSF were determined with high performance liquid chromatography (ECD-300, Eicom Corporation, Kyoto, Japan). All results are presented as the mean (SD), and Kruskal-Wallis tests were used to analyze the differences in the data among controls and patients with WS and CwG.

\section{Results}

3.1. Pathological Analysis in the PPN. The numbers of MAP2-immunoreactive neurons and AchNs immunoreactive for AchE were reduced significantly in WS/LGS and WS cases (Table 3 and Figures 1(a) and 1(b)). The percentage of AchNs in those cases seemed to be fewer than that of controls, but the difference was not significant. There was no relationship between the etiology and the reduction of neurons (data not shown). In contrast, there were no significant differences between controls and WS cases in the number and percentages of either CANs or GABAi, which were immunoreactive for $\mathrm{TH}$ or $\mathrm{CD}$, respectively (Table 3 and Figures 1(c) and 1(d)). DRPLA cases showed a significant reduction in the number and percentage of AchNs $(P<0.01)$, although there was no significant difference in the number of MAP2-immunoreactive neurons. There were no changes in the numbers or percentages of either CANs or GABAi (Figure 2). There were no increases in GFAPimmunoreactive astrocytes in WS/LGS, WS, or DRPLA cases (data not shown).

3.2. CSF Analysis. WS patients demonstrated a significant reduction in the levels of inhibitory neuropeptides (glycine and GABA) but not of those of acetylcholine, excitatory amino acids, or monoamine metabolites (Table 4). Such reductions in the CSF levels of inhibitory neuropeptides had no relationship with either disease type or prognosis (data not shown). In contrast, $\mathrm{CwG}$ patients showed a significant increase in the CSF levels of acetylcholine and a significant decrease in the CSF levels of 5-HIAA, which is a serotonin metabolite, whereas there were no changes in those of either amino acids or CAN metabolites (Table 4).

\section{Discussion}

Mutations in the neuronal nAChR subunit genes CHRNA4, CHRNB2, and probably CHRNA2 have been demonstrated to cause ADNFLE [17]. Inasmuch as neuronal nAChRs 
TABLE 3: Results of the quantitative analysis in the pedunculopontine tegmental nucleus in cases with a history of West syndrome.

\begin{tabular}{lcccccccc}
\hline & Age & MAP2 & AchE & \%AchE & TH & \%TH & CD & $\%$ CD \\
\hline Controls $(n=6)$ & $4 \mathrm{~m}-38 \mathrm{y}$ & $243(41)$ & $68(16)$ & $29(9)$ & $25(13)$ & $10(5)$ & $27(17)$ & $11(6)$ \\
WS/LGS $(n=6)$ & $5-18 \mathrm{y}$ & $181(39)$ & $12(15)$ & $8(12)$ & $35(10)$ & $19(5)$ & $18(20)$ & $9(10)$ \\
WS $(n=6)$ & $5-26 \mathrm{y}$ & $184(38)$ & $29(39)$ & $13(18)$ & $27(18)$ & $16(10)$ & $31(14)$ & $17(8)$ \\
Kruskal-Wallis & & $P<0.05$ & $P<0.05$ & & & &
\end{tabular}

"WS/LGS" and "WS" denote cases with a history of West syndrome (WS) and Lennox-Gastaut syndrome (LGS) and cases with a history of only WS, respectively. All results are presented as the mean (SD), and both the total number of neurons that were immunoreactive for each marker and the percentage of neurons immunoreactive for each marker relative to the total neurons are shown. m: months; y: years; MAP2: microtubule-associated protein 2; AchE: acetylcholinesterase; TH: tyrosine hydroxylase; CD: calbindin-D28K.

TABLE 4: Summary of the data in the analysis of the cerebrospinal fluid.

\begin{tabular}{|c|c|c|c|c|c|c|c|c|c|}
\hline & Age & $\begin{array}{c}\text { Acetylcholine } \\
(\mathrm{fmol})\end{array}$ & $\begin{array}{c}\text { Aspartate } \\
(\mathrm{pmol})\end{array}$ & $\begin{array}{c}\text { Glutamate } \\
(\mathrm{pmol})\end{array}$ & $\begin{array}{l}\text { Glycine } \\
\text { (pmol) }\end{array}$ & $\begin{array}{c}\text { GABA } \\
(\mathrm{pg} / \mu \mathrm{L})\end{array}$ & $\begin{array}{l}\text { MHPG } \\
(\mathrm{pg} / \mu \mathrm{L})\end{array}$ & $\begin{array}{c}\text { HVA } \\
(\mathrm{pg} / \mu \mathrm{L})\end{array}$ & $\begin{array}{c}\text { 5-HIAA } \\
(\mathrm{pg} / \mu \mathrm{L})\end{array}$ \\
\hline Controls $(n=5)$ & $0-6 \mathrm{~m}$ & $1.8(1.2)$ & $305(179)$ & $94(39)$ & $477(214)$ & $44(13)$ & $46(33)$ & $204(133)$ & $912(520)$ \\
\hline West syndrome $(n=3)$ & $5-8 \mathrm{~m}$ & $5.8(4.3)$ & $81(25)$ & $37(7)$ & $137(49)$ & $22(7)$ & $77(24)$ & $203(64)$ & $698(225)$ \\
\hline $\mathrm{CwG}(n=5)$ & $8 \mathrm{~m}-2 \mathrm{y}$ & $231(199)$ & $223(108)$ & $116(50)$ & $408(105)$ & $70(27)$ & $43(12)$ & $86(21)$ & $36(13)$ \\
\hline Kruskal-Wallis & & $P<0.01$ & & & $P<0.05$ & $P<0.05$ & & & $P<0.01$ \\
\hline
\end{tabular}

All results are presented as the mean (SD). m: months; y: years; MHPG: 3-methoxy-4-hydroxyphenylethylglycol; HVA: homovanillic acid; 5-HIAA: 5hydroxyindoleacetic acid; CwG: benign convulsions associated with mild gastroenteritis.

participate in synaptic plasticity and are involved in learning, memory, and development, disruptions or alterations of nicotinic cholinergic mechanisms lead to nervous diseases, including Alzheimer's disease, autism, and epilepsy [18]. The thalamus and the cortex are innervated by cholinergic neurons projecting from the brainstem, and an imbalance between excitation and inhibition in the mutation of nAChRs may generate epileptic seizures by disturbing the thalamocortical circuits [19]. Of interest, lamotrigine, which is an anticonvulsive drug that is effective for the treatment of various epileptic syndromes, has been shown to block $\alpha 4 \beta 2$ nAChRs-mediated currents but to not affect glutamate- or GABA-induced currents in rat dopaminergic neurons [20]. These findings strongly indicate the significance of lesions of AchNs in epileptogenesis. The morphological identification of the neuronal nAChR remains to be elaborated in autopsy specimens, and we performed an immunohistochemical analysis on the lesions of AchNs themselves in the brains, in addition to the preliminary determination of the levels of acetylcholine, neuropeptides, and monoamine metabolites in the CSF.

The cases of WS/LGS and WS had a reduction of AchNs in addition to decreases in total neurons in the PPN (Table 3). However, the DRPLA cases showed a reduction in the number and percentage of AchNs in the preservation of total neurons (Figure 2). Similarly, in our previous immunohistochemical study on CANs in the brainstem, WS/LGS cases demonstrated a reduction in the levels of expression of TH and tryptophan hydroxylase [6], whereas such expression was preserved comparatively well in DRPLA cases [7]. It is likely that the differences in the lesions of AchNs and CANs in the brainstem may be related to the generation of specific phenotypes of convulsions in each epileptic syndrome. Intriguingly, the absence of gliosis and the comparative preservation of CANs and GABAis were commonly observed in the PPN in WS/LGS, WS, and DRPLA cases. In contrast, cases of perinatal brain damage showed a reduction in the percentage of AchNs and a compensatory increase in the percentage of CANs in addition to gliosis [11]. It is well known that cases with a history of WS and DRPLA cases exhibited small tegmentums, especially in the pons $[21,22]$. These findings indicate that the reduction of AchNs is a specific change and may not be caused by destructive lesioning of the PPN itself. Some authors have speculated that WS may result from temporal desynchronization of several CNS developmental processes [23]. Accumulation of mutant DRPLA protein also involves a wide range of CNS regions far beyond the systems previously reported to be affected [24]. Because the PPN has afferent and efferent connections to both cortical and subcortical structures, selective lesioning of AchNs is likely to be involved in epileptogenesis in WS, LGS, and DRPLA.

The Prader-Willi syndrome case showed a reduction of AchNs in the PPN but not in the NbM [12], whereas cases of xeroderma pigmentosum group A had such reductions in both nuclei [13], suggesting the presence of different regional patterns in the impairment of AchNs. Although Tsuchiya et al. reported the absence of significant neuronal loss in the $\mathrm{NbM}$ in DRPLA cases [25], further immunohistochemical evaluations are needed in the NbM in cases of WS, LGS, and DRPLA.

The CSF levels of neurotransmitters, neuropeptides, and their metabolites have been examined in patients with refractory epilepsy, for example, WS patients tend to show changes that are related to an impairment of serotonergic neurons and GABAis [26], and DRPLA patients demonstrate an age-related increase of amine precursors (tryptophan and tyrosine) [27]. However, the results are heterogeneous and variable, which is probably due to differences in etiology, analysis systems, and the selection of control samples. More 


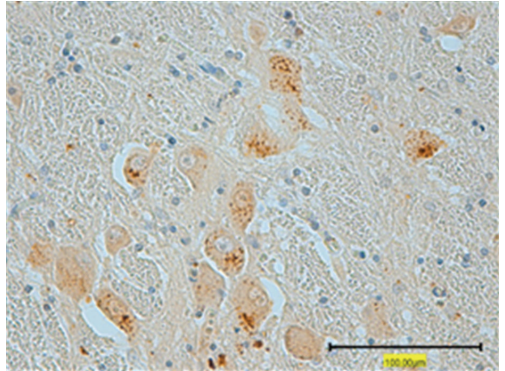

(a)

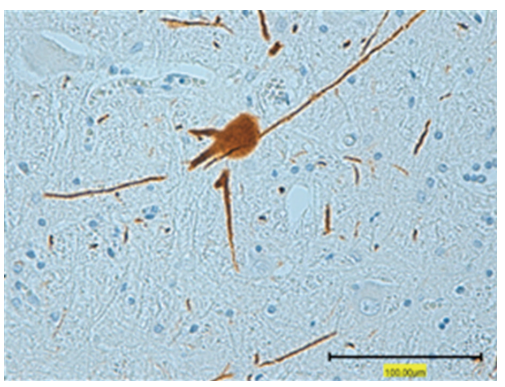

(c)

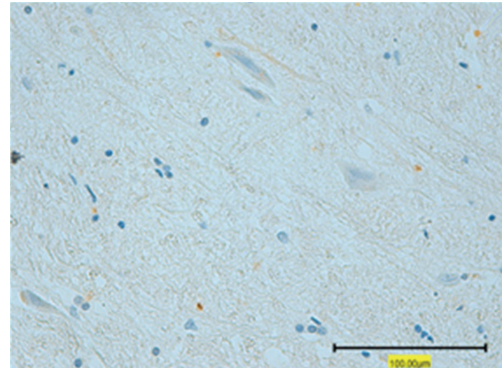

(b)

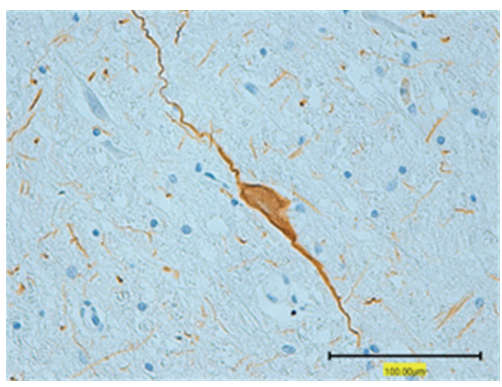

(d)

FIGURE 1: Representative illustrations of immunohistochemistry in the pedunculopontine tegmental nucleus. Photographs (a) and (c) are from a 22-year-old control who died of disseminated intravascular coagulation syndrome. Photographs (b) and (d) are from a 21-year-old case of lissencephaly with a history of West syndrome and Lennox-Gastaut syndrome. In the immunohistochemistry for acetylcholinesterase, the control showed many immunoreactive neurons (a), which were reduced in case (b). In the immunohistochemistry for tyrosine hydroxylase, immunoreactive neurons with neuronal processes were identified commonly in the control $(\mathrm{c})$ and the case $(\mathrm{d})$. Bars $=100 \mu \mathrm{m}$.

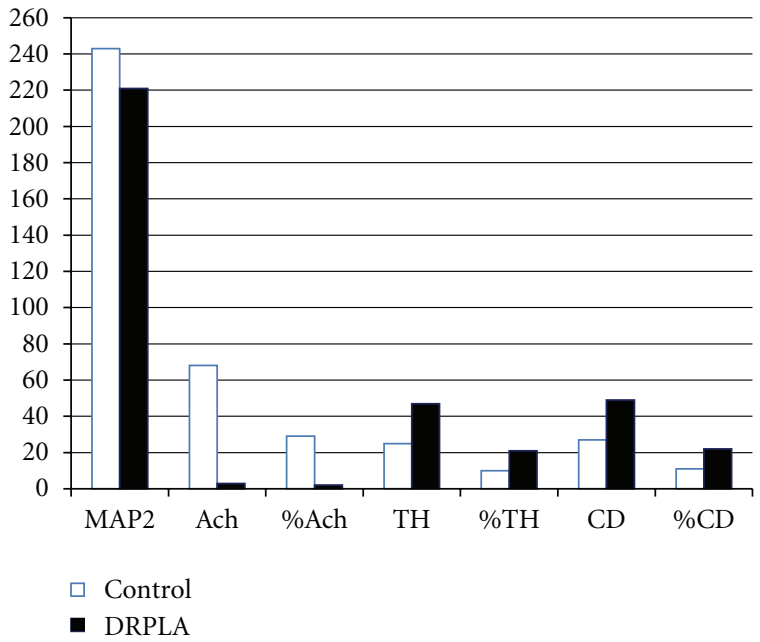

FIGURe 2: Data of the quantitative analysis in the pedunculopontine tegmental nucleus of cases with dentatorubral-pallidoluysian atrophy (DRPLA). Open and closed columns denote the numbers and percentages of each marker in controls and DRPLA cases, respectively. M: months; y: years; MAP2: microtubule-associated protein 2; AchE: acetylcholinesterase; TH: tyrosine hydroxylase; CD: calbindin-D28K.

recent and elaborate analyses in patients with early-onset severe epileptic encephalopathy failed to identify changes in the CSF levels of monoamine metabolites [28]. The CSF levels of acetylcholine were reported to be reduced in patients with PME, although that has rarely been examined [29].

In our preliminary CSF analysis, we examined 3 WS infants just before treatment and CwG children as disease controls, but not patients with DRPLA, in which the CSF samples were not acquired. It is interesting that $3 \mathrm{WS}$ patients demonstrated changes in the CSF levels of glycine and GABA, whereas there were no significant differences in those of excitatory neuropeptides, acetylcholine, and monoamine metabolites (Table 4). The reduced levels of GABA seem to be in accordance with the findings in a previous report [26]. In order to obtain a definite conclusion, it is required to increase the number of patients and pay careful consideration to differences in etiology and prognosis. However, it is surprising that $5 \mathrm{CwG}$ patients showed a significant change in the CSF levels of acetylcholine and serotonin metabolites (Table 4). Generally, seizure and developmental prognosis is good in patients with $\mathrm{CwG}$, but the detailed pathogenesis has not been clarified yet $[16,30]$. It is possible that transient alterations of neurotransmitter metabolism are involved in CwG.

In conclusion, the pathological study and preliminary CSF analysis suggested the possible involvement of lesions of AchNs in the pathogenesis of WS/LGS, DRPLA, and CwG. The selective reduction of AchNs was verified in the PPN in WS and DRPLA. In order to reach a definite conclusion, we should perform similar immunohistochemical examinations in cases with PME other than DRPLA and should expand the number of patients in the CSF analysis. 


\section{Conflict of Interests}

None of the authors has any conflicts of interests to disclose.

\section{References}

[1] O. Dulac and I. Tuxhorn, "Infantile spasms and West syndrome," in Epileptic Syndrome in Infancy, Childhood and Adolescence, J. Roger, M. Bureau, C. H. Genton, P. Genton, C. A. Tassinari, and P. Wolf, Eds., pp. 53-72, John Libbey Eurotext, Montrouge, France, 2005.

[2] T. Tsuji, A. Okumura, H. Ozawa, M. Ito, and K. Watanabe, "Current treatment of West syndrome in Japan," Journal of Child Neurology, vol. 22, no. 5, pp. 560-564, 2007.

[3] A. Shahwan, M. Farrell, and N. Delanty, "Progressive myoclonic epilepsies: a review of genetic and therapeutic aspects," The Lancet Neurology, vol. 4, no. 4, pp. 239-248, 2005.

[4] M. Yamada, S. Tsuji, and H. Takahashi, "Genotype-phenotype correlation in CAG-repeat diseases," Neuropathology, vol. 22, no. 4, pp. 317-322, 2002.

[5] R. Miyata, M. Hayashi, N. Tanuma, K. Shioda, R. Fukatsu, and S. Mizutani, "Oxidative stress in neurodegeneration in dentatorubral-pallidoluysian atrophy," Journal of the Neurological Sciences, vol. 264, no. 1-2, pp. 133-139, 2008.

[6] M. Hayashi, "Neuropathology of the limbic system and brainstem in West syndrome," Brain and Development, vol. 23, no. 7, pp. 516-522, 2001.

[7] M. Hayashi, S. Kumada, K. Shioda, and R. Fukatsu, "Neuropathological analysis of the brainstem and cerebral cortex lesions on epileptogenesis in hereditary dentatorubral-pallidoluysian atrophy," Brain and Development, vol. 29, no. 8, pp. 473-481, 2007.

[8] O. Isacson, H. Seo, L. Lin, D. Albeck, and A. C. Granholm, "Alzheimer's disease and Down's syndrome: roles of APP, trophic factors and ACh," Trends in Neurosciences, vol. 25, no. 2, pp. 79-84, 2002.

[9] J. Berger-Sweeney, "The cholinergic basal forebrain system during development and its influence on cognitive processes: important questions and potential answers," Neuroscience and Biobehavioral Reviews, vol. 27, no. 4, pp. 401-411, 2003.

[10] M. S. Lee, J. O. Rinne, and C. D. Marsden, "The pedunculopontine nucleus: its role in the genesis of movement disorders," Yonsei Medical Journal, vol. 41, no. 2, pp. 167-184, 2000.

[11] Y. Anzai, M. Hayashi, T. Ohya, and S. Yokota, "The pedunculopontine nucleus in developmental disorders of the basal ganglia," Neuropathology, vol. 28, no. 3, pp. 258-263, 2008.

[12] M. Hayashi, R. Miyata, and N. Tanuma, "Decrease in acetylcholinergic neurons in the pedunculopontine tegmental nucleus in a patient with Prader-Willi syndrome," Neuropathology, vol. 31, no. 3, pp. 280-285, 2011.

[13] M. Hayashi, T. Ohto, K. Shioda, and R. Fukatsu, "Lesions of cortical GABAergic interneurons and acetylcholine neurons in xeroderma pigmentosum group A," Brain and Development, vol. 34, no. 4, pp. 287-292, 2012.

[14] R. C. Hogg, M. Raggenbass, and D. Bertrand, "Nicotinic acetylcholine receptors: from structure to brain function," Reviews of Physiology, Biochemistry and Pharmacology, vol. 147, pp. 1-46, 2003.

[15] P. Ryvlin, S. Rheims, and G. Risse, "Nocturnal frontal lobe epilepsy," Epilepsia, vol. 47, supplement S2, pp. 83-86, 2006.

[16] A. Okumura, N. Uemura, T. Negoro, and K. Watanabe, "Efficacy of antiepileptic drugs in patients with benign convulsions with mild gastroenteritis," Brain and Development, vol. 26, no. 3, pp. 164-167, 2004.

[17] J.-C. Hoda, W. Gu, M. Friedli et al., "Human nocturnal frontal lobe epilepsy: pharmocogenomic profiles of pathogenic nicotinic acetylcholine receptor $\beta$-subunit mutations outside the ion channel pore," Molecular Pharmacology, vol. 74, no. 2, pp. 379-391, 2008.

[18] J. A. Dani and D. Bertrand, "Nicotinic acetylcholine receptors and nicotinic cholinergic mechanisms of the central nervous system," Annual Review of Pharmacology and Toxicology, vol. 47, pp. 699-729, 2007.

[19] M. Raggenbass and D. Bertrand, "Nicotinic receptors in circuit excitability and epilepsy," Journal of Neurobiology, vol. 53, no. 4, pp. 580-589, 2002.

[20] C. Zheng, K. Yang, Q. Liu et al., "The anticonvulsive drug lamotrigine blocks neuronal $\alpha 4 \beta 2$ nicotinic acetylcholine receptors," Journal of Pharmacology and Experimental Therapeutics, vol. 335, no. 2, pp. 401-408, 2010.

[21] J. Satoh, T. Mizutani, and Y. Morimatsu, "Neuropathology of the brainstem in age-dependent epileptic encephalopathyespecially of cases with infantile spasms," Brain and Development, vol. 8, no. 4, pp. 443-449, 1986.

[22] S. Oyanagi, "Hereditary dentatorubral-pallidoluysian atrophy," Neuropathology, vol. 20, supplement, pp. S42-S46, 2000.

[23] J. D. Frost Jr. and R. A. Hrachovy, "Pathogenesis of infantile spasms: a model based on developmental desynchronization," Journal of Clinical Neurophysiology, vol. 22, no. 1, pp. 25-36, 2005.

[24] S. Tsuji, "Dentatorubral-pallidoluysian atrophy," Handbook of Clinical Neurology, vol. 103, pp. 587-594, 2012.

[25] K. Tsuchiya, S. Oyanagi, K. Arima et al., "Dentatorubropallidoluysian atrophy: clinicopathological study of dementia and involvement of the nucleus basalis of Meynert in seven autopsy cases," Acta Neuropathologica, vol. 96, no. 5, pp. 502-508, 1998.

[26] M. R. Pranzatelli, "Putative neurotransmitter abnormalities in infantile spasms: cerebrospinal fluid neurochemistry and drug effects," Journal of Child Neurology, vol. 9, no. 2, pp. 119-129, 1994.

[27] Y. Takeuchi, H. Matsushita, H. Sakai, H. Kawano, and M. Ochi, "Developmental changes in cerebrospinal fluid concentrations of monoamine-related substances in patients with dentatorubral-pallidoluysian atrophy," Journal of Child Neurology, vol. 16, no. 2, pp. 79-82, 2001.

[28] B. Echenne, A. Roubertie, J. Leydet, F. Rivier, and G. F. Hoffmann, "Monoamine metabolism study in severe, early-onset epilepsy in childhood," Epileptic Disorders, vol. 10, no. 2, pp. 130-135, 2008.

[29] M. R. Pranzatelli, I. Hanin, E. Tate et al., "Cerebrospinal fluid free choline in movement disorders of paediatric onset," European Journal of Paediatric Neurology, vol. 2, no. 1, pp. 33-39, 1998.

[30] S. Yamashita, A. Okumura, T. Yamamoto, K. Shimojima, T. Tanabe, and T. Shimizu, "SCN1B is not related to benign partial epilepsy in infancy or convulsions with gastroenteritis," Neuropediatrics, vol. 42, no. 4, pp. 135-137, 2011. 


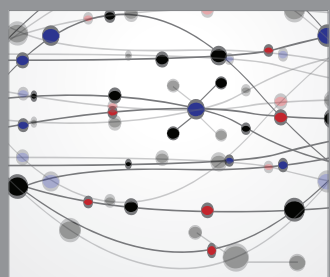

The Scientific World Journal
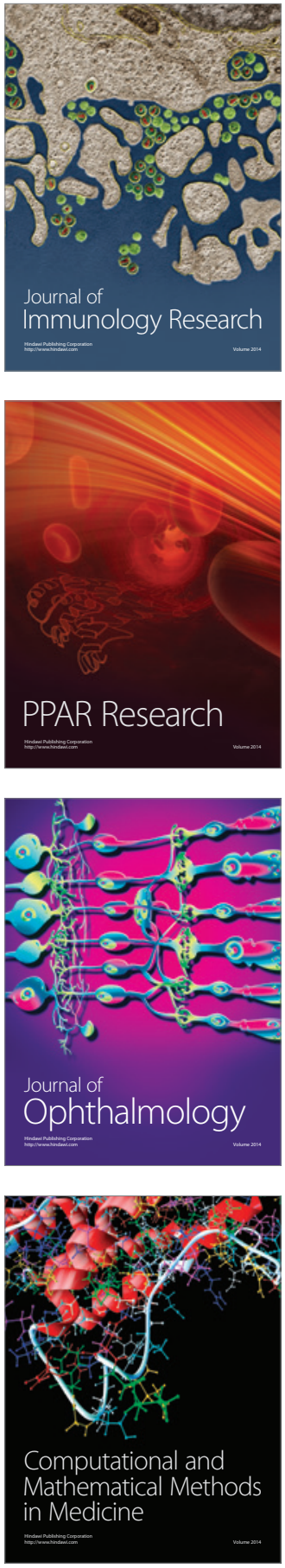

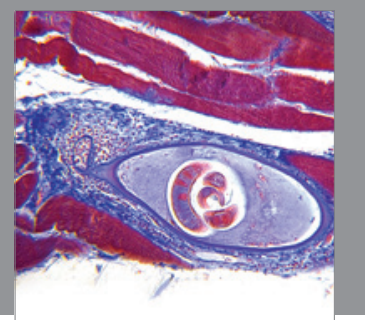

Gastroenterology

Research and Practice
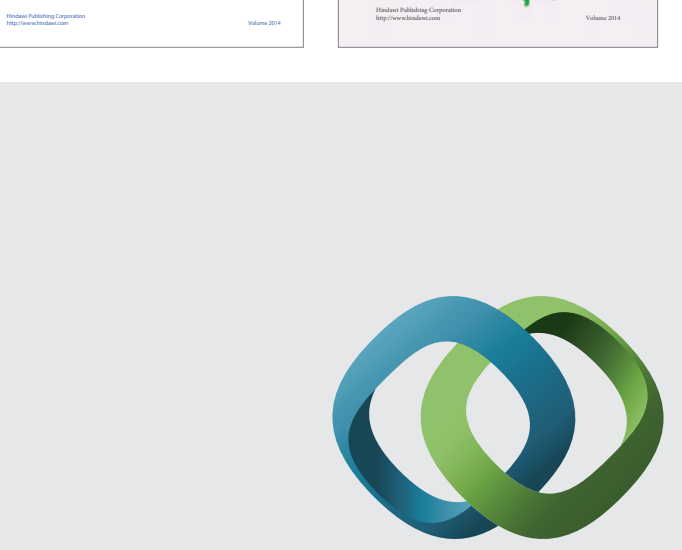

\section{Hindawi}

Submit your manuscripts at

http://www.hindawi.com
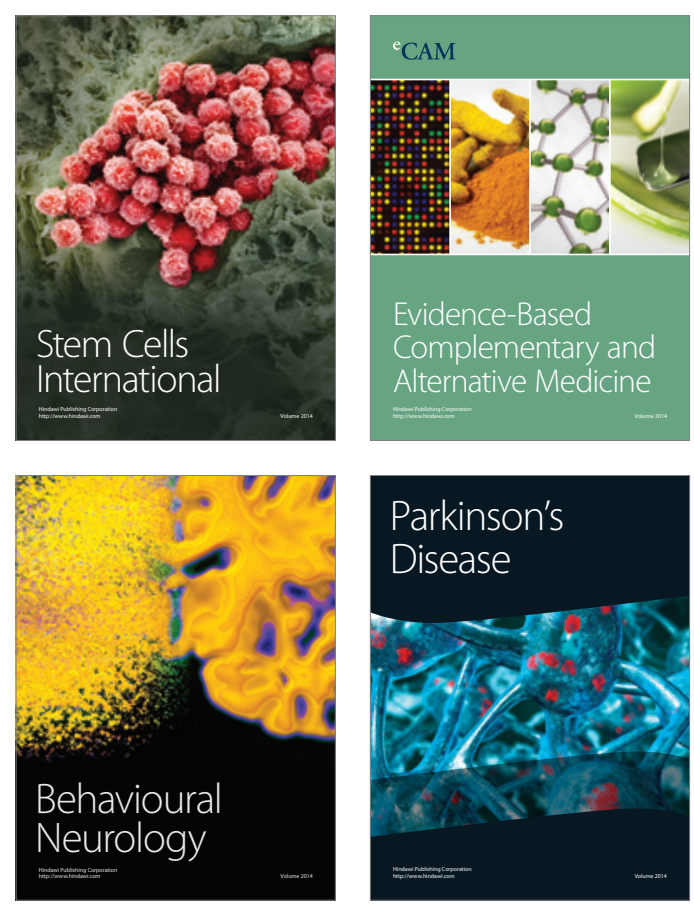

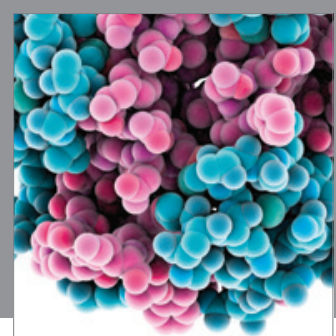

Journal of
Diabetes Research

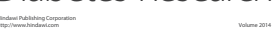

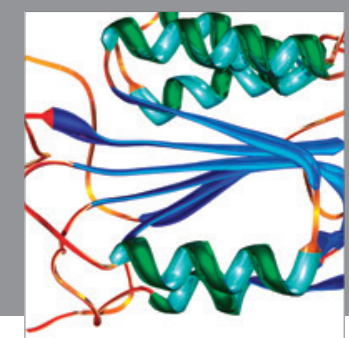

Disease Markers
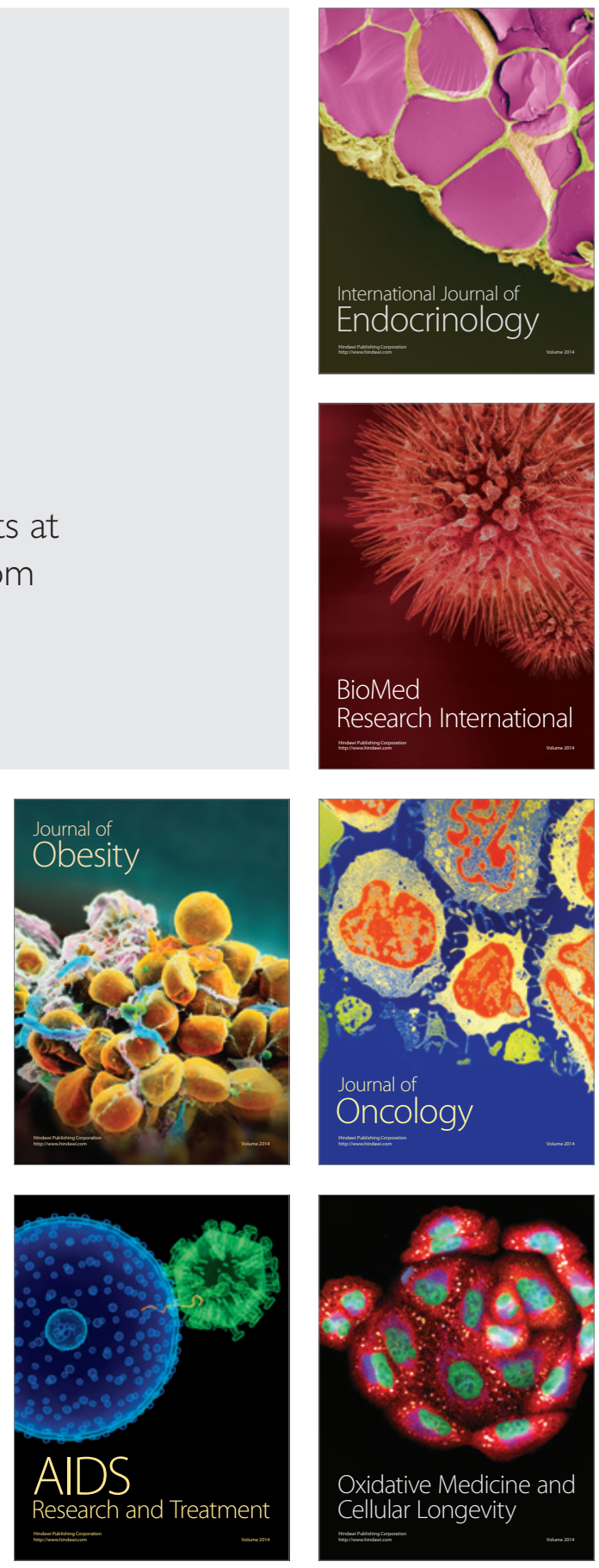